SUBMitTed to ApJ

Preprint typeset using $\mathrm{LAT}_{\mathrm{EX}}$ style emulateapj v. 14/09/00

\title{
HEATING, CONDUCTION AND MINIMUM TEMPERATURES IN COOLING FLOWS
}

\author{
Mateusz Ruszkowski and Mitchell C. Begelman ${ }^{1}$ \\ JILA, Campus Box 440, University of Colorado, Boulder CO 80309-0440 \\ mr@quixote.colorado.edu; mitch@jila.colorado.edu \\ submitted to ApJ
}

\begin{abstract}
There is mounting observational evidence from Chandra for strong interaction between keV gas and AGN in cooling flows. It is now widely accepted that the temperatures of cluster cores are maintained at a level of $\sim 1 \mathrm{keV}$ and that the mass deposition rates are lower than earlier ROSAT/Einstein values. Recent theoretical results suggest that thermal conduction can be very efficient even in magnetized plasmas. Motivated by these discoveries, we consider a "double heating model" which incorporates the effects of simultaneous heating by both the central AGN and thermal conduction from the hot outer layers of clusters. Using hydrodynamical simulations, we demonstrate that there exists a family of solutions that does not suffer from the cooling catastrophe. In these cases, clusters relax to a stable final state, which is characterized by minimum temperatures of order $1 \mathrm{keV}$ and density and temperature profiles consistent with observations. Moreover, the accretion rates are much reduced, thereby reducing the need for excessive mass deposition rates required by the standard cooling flow models.
\end{abstract}

Subject headings: galaxies: clusters — cooling flows - X-rays: galaxies — conduction — intergalactic medium

\section{INTRODUCTION}

Radiative cooling of gas in the central regions of galaxy clusters occurs on a timescale much shorter than the Hubble time. The cooling time-scale of this gas increases with distance from the cluster core. In the absence of any heating sources, this implies that the intracluster medium must accrete subsonically toward the center in order to maintain pressure equilibrium with the gas at larger radii. The mass deposition rates predicted by this cooling flow model are very high and range typically from 10 to 1000 solar masses per year. X-ray observations made prior to the launch of the Chandra Observatory seemed to be broadly consistent with this picture (Fabian 1994). Although both the gas temperature and cooling time are observed to decline toward cluster cores, new Chandra and XMM-Newton observations show a remarkable lack of emission lines from gas at temperatures below $\sim 1 \mathrm{keV}$ in the central regions of clusters (Peterson et al. 2001; Allen et al. 2001). Moreover, the cooling mass deposition rates obtained with Chandra and $X M M$ using spectroscopic methods are $\sim 10$ times smaller than earlier estimates based on ROSAT and Einstein observations (McNamara et al. 2001; David et al. 2001; Peterson et al. 2001). On the other hand, morphological cooling rates give accretion rates vastly exceeding the ones based on spectroscopic methods (David et al. 2001). The strong discrepancy between these results indicates either that the gas is prevented from cooling by some heating process or that it cools without any spectroscopic signatures (Fabian 2001).

In this paper, we consider cooling flow models that incorporate the effects of heating by central active galactic nuclei and thermal conduction from the hot outer layers of clusters. We show that evolving density and temperature profiles can relax to stable final states, which are consis- tent with X-ray observations. Equilibria are characterized by minimum temperatures of order $1 \mathrm{keV}$ at radii $\sim 1 \mathrm{kpc}$. The paper is organized as follows. In section 2 we discuss the observational and theoretical motivation for including both AGN heating and thermal conduction. In section 3 we present the details of our model. The results of hydrodynamic simulations are presented in Section 4 and the main results are summarized in section 5 .

\section{HEATING MECHANISMS}

\subsection{Feedback and heating by $A G N$}

A large fraction $(\sim 70 \%)$ of $\mathrm{cD}$ galaxies in the centers of cooling flow clusters shows evidence for powerful radio activity. This fraction is much larger than in non-cooling flow clusters and suggests a link between the presence of the cooling gas and the activity of the central supermassive black hole. Currently, Chandra offers the best opportunity to study this coupling as the cluster cores are optically thin and can be spatially resolved down to scales of a few kpc within $z<0.1$. These scales are comparable to the sizes of the radio sources. Recent Chandra observations of the Perseus, Hydra A and many other clusters reveal holes in the X-ray surface brightness which coincide with radio lobes (Fabian 2001; McNamara et al. 2001). These radio sources inflate bubbles of hot plasma that subsequently rise through the cluster atmosphere, mixing and heating it up. This mechanism has been invoked to explain the reduced cooling rates (Jones et al. 2002; Churazov et al. 2002).

\subsection{Heating by thermal conduction}

A potential difficulty with reducing the cooling accretion rate by means of a central heating source is that too strong a heating source will lead to very strong convection, which could remove metallicity gradients observed

${ }^{1}$ Also at Department of Astrophysical and Planetary Sciences, University of Colorado 
by Chandra in cluster cores (Johnstone at al. 2002). Therefore, the limits on energy requirements of central AGN and on the amount of mixing and convection suggest that an additional heating source is required to offset cooling rates. An important candidate is thermal conduction of heat from the outer hot layers of the cooling flow cluster. With some exceptions (Bertschinger and Meiksin 1986; Malyshkin 2001; Brighenti and Mathews 2002; $\quad$ Voigt et al. 2002; Fabian et al. 2002) this effect has been largely neglected in observational and theoretical studies due to the preconception that the conduction coefficient would be suppressed much below the classical Spitzer value by magnetic fields. Indeed, there is strong evidence that clusters are magnetized by intermittent radio galaxies in cluster cores (Clarke et al. 2001; Kronberg et al. 2001; Reynolds and Begelman 1997). However, recent theoretical work by (Narayan and Medvedev 2001) suggests that if the magnetic field is highly turbulent, then thermal conduction is relatively efficient. They find that if the turbulence spectrum extends over two or more decades in wave vector, then the thermal conduction coefficient is only a factor $\sim$ a few below the Spitzer value and thus could play a significant role in cooling flows in clusters of galaxies. The other argument in favor of thermal conduction is that it is a very strongly increasing function of temperature and, therefore, could be efficient in the outer layers of clusters where temperatures are high.

\subsection{Simultaneous heating by $A G N$ and conduction}

If radiative cooling is balanced solely by energy input from a central supermassive black hole then a cooling flow can be quenched provided that a sufficient amount of power is provided in kinetic form (McNamara 2002). Models based on the assumption that radiative cooling is balanced by energy input from AGN predict that the mechanical power of AGN in cooling flows is much higher than the presently observed bolometric luminosities of these objects (Churazov et al. 2002). However, the power necessary to offset a cooling flow may still be present in the kinetic form after the central AGN becomes inactive. In fact, the required power is consistent with estimates of jet power in some objects. Theoretical models of impulsive central heating in elliptical galaxies (Binney and Tabor 1995) predict violent successive cooling catastrophes and temperature rising towards the center for radii less than $\sim 100 \mathrm{kpc}$. On the other hand, attempts to build cooling flow models with conduction as the only heating mechanism fail. Such models predict that the cooling time of the central cluster region diminishes to less than the free-fall time, leading to a cooling catastrophe (Meiksin 1988). This results in supersonic accretion of cold and dense material in the core, which is contrary to observations. Stable models can be obtained but they assume a significant amount of distributed mass dropout which does not have observational grounding. Moreover, even when the models are stable, such cooling flows suffer from additional problems. Due to the strong dependence of the conduction coefficient on temperature $\left(\propto T^{5 / 2}\right)$, the predicted temperatures either drop by less than a factor of 2 or the central temperatures are so low that heat conduction is negligible and the central accretion rate often becomes very high. This suggests that heat conduction alone cannot substantially reduce mass accretion rates everywhere within a cluster. Recently, (Brighenti and Mathews 2002) considered centrally heated cooling flow models with conduction. They concluded that such models are grossly incompatible with observations and were unable to find even a single acceptable cooling flow model using their heating prescriptions.

In the next section we present details of our model and look at the consequences of bridging these two heating regimes.

\section{3. "DOUble heAting" MOdEL}

\subsection{Physical assumptions}

We solve numerically the equations of hydrodynamics in the following form

$$
\begin{aligned}
\frac{\partial \rho}{\partial t}+\nabla \cdot(\rho \mathbf{v})= & 0 \\
\frac{\partial \mathbf{S}}{\partial t}+\nabla \cdot(\mathbf{S v})= & -\nabla p-\rho \nabla \Psi \\
\frac{\partial e}{\partial t}+\nabla \cdot(e \mathbf{v})= & -p \nabla \cdot \mathbf{v}-\nabla \cdot \mathbf{F}_{\mathrm{cond}}-\nabla \cdot \mathbf{F}_{\mathrm{conv}} \\
& -n_{e}^{2} \Lambda(T)+\mathcal{H},
\end{aligned}
$$

where $\rho$ is the mass density, $n_{e}$ the electron number density, $p$ the pressure, $\mathbf{v}$ the velocity, $\mathbf{S}=\rho \mathbf{v}$ the momentum vector, $e$ the internal energy density, $\Psi$ the gravitational potential, $\Lambda(T)$ the cooling function and $\mathcal{H}$ the heating rate per unit volume. We adopt an equation of state $p=(\gamma-1) e$ and consider models with $\gamma=5 / 3$. The conductive flux $\mathbf{F}_{\text {cond }}$ is given by

$$
\mathbf{F}_{\text {cond }}=-f \kappa T^{5 / 2} \nabla T,
$$

where $\kappa$ is the Spitzer conductivity

$$
\kappa=\frac{1.84 \times 10^{-5} T^{5 / 2}}{\ln \lambda},
$$

with the Coulomb logarithm $\ln \lambda=37$, and $f$ is a reduction factor $(0 \leq f \leq 1)$. The saturation of the conductive flux was not important for the parameters considered in this paper. The convective flux $\mathbf{F}_{\text {conv }}$ is given by mixing length theory

$$
\mathbf{F}_{\text {conv }}=\left\{\begin{array}{ll}
\frac{1}{2^{5 / 2} c_{p}} g^{1 / 2} \rho l_{m}^{2}(-\nabla \hat{s})^{3 / 2} & \text { if } \nabla \hat{s}<0 \\
0 & \text { otherwise }
\end{array},\right.
$$

where $g$ is the gravitational acceleration, $l_{m}$ is the mixing length, $\hat{s}=c_{v} \ln \left[(\gamma-1) e / \rho^{\gamma}\right]$ is the gas entropy, $c_{v}$ is the specific heat per unit volume, $c_{p}=\gamma k_{B} /\left[(\gamma-1) \mu m_{p}\right]$ is the specific heat per unit mass at constant pressure, $\mu=0.5$ is the mean molecular weight and $\gamma$ is the adiabatic index. In mixing length theory $l_{m}$ is a free parameter. We use $l_{m}=\min [0.3(\gamma-1) e /(\rho g), r]$, where $r$ is the distance from the cluster center. Motivated by recent Chandra X-ray observations of clusters (e.g., (Schmidt et al. 2001; Allen et al. 2001)), we parameterize the dark matter distribution using a non-singular isothermal mass density profile. We add a contribution from the central galaxy also in the form of a non-singular isothermal profile but characterized by different values of parameters. 
We assume that the total potential does not evolve. The contribution of the gas to gravity is negligible throughout the simulation volume. The corresponding total gravitational potential is then given by $\Psi_{\text {tot }}=\Psi_{c}+\Psi_{g}$, where

$\Psi_{c, g}=\sigma_{c, g}^{2} \ln \left[1+\left(\frac{r}{r_{c, g}}\right)^{2}\right]+2 \sigma_{c, g}^{2}\left(\frac{r_{c, g}}{r}\right) \arctan \left(\frac{r}{r_{c, g}}\right)$,

where $\sigma_{c, g}$ is the cluster or galactic velocity dispersion and $r_{c, g}$ is the core radius of the cluster or galaxy.

Note that equations (1)-(3) do not include terms related to mass dropout rate. Such terms are usually invoked to avoid a cooling catastrophe by removing cooled gas from the flow. Thermal instabilities, which lead to mass dropout, appear when the cooling time is comparable to or shorter than the dynamical time. In our simulations the cooling time is longer by a factor $10-100$ than the dynamical time, so our assumption is justified. A small amount of mass dropout may still occur in the central regions if the gas becomes locally overdense, e.g., due to interactions of the central AGN with the surrounding medium. However, as mentioned above, there is no strong observational indication that significant amounts of gas are decoupling throughout the cooling flow region, although star-forming regions and small amounts of cool gas in the form of $\mathrm{H \alpha}$ emission have been detected in the innermost regions of clusters. Such colder gas also could have been lifted from very small radii to larger distances by the central AGN.

\subsubsection{Heating and cooling}

Following (Tozzi and Norman 2001) we use an approximation to the cooling function based on detailed calculations by (Sutherland and Dopita 1993)

$$
n_{e}^{2} \Lambda=\left[C_{1}\left(k_{B} T\right)^{\alpha}+C_{2}\left(k_{B} T\right)^{\beta}+C_{3}\right] n_{i} n_{e},
$$

where $n_{i}$ is the ion number density and the units for $k_{B} T$ are $k e V$. For an average metallicity $Z=0.3 Z_{\odot}$ the constants in equation (8) are $\alpha=-1.7, \beta=0.5$, $C_{1}=8.6 \times 10^{-3}, C_{2}=5.8 \times 10^{-2}$ and $C_{3}=6.4 \times 10^{-2}$ and we can approximate $n_{i} n_{e}=0.704\left(\rho / m_{p}\right)^{2}$. The units of $\Lambda$ are $10^{-22} \mathrm{erg} \mathrm{cm}^{3} \mathrm{~s}^{-1}$. This cooling function incorporates the effects of free-free and line cooling.

The final term in equation (3) represents heating by the central AGN. Since it is ultimately heating by mechanical energy and particles from radio sources, it will be distributed in radius. We adopt a physically motivated "effervescent heating" mechanism (Begelman 2001) to describe the volume heating rate. The physical motivation for this model is as follows. Suppose the central radio source deposits buoyant gas which distributes itself relatively evenly among bubbles or filaments but does not mix microscopically with the intracluster medium (ICM). These bubbles will then rise through the ICM and expand because of the non-negligible pressure gradient. Bubble expansion will be associated with the conversion of the internal bubble energy to kinetic form and, eventually, to heat due to disorganized motion of the ICM. Since the bubbles rise on a timescale shorter than the radiative cooling time, the mechanism should reach a quasi-steady state and the dependence on details of bubble filling factor, rise rate, etc. cancel out. In a steady state (and assuming spherical symmetry), the energy flux available for heating is then

$$
\dot{e} \propto p_{b}(r)^{\left(\gamma_{b}-1\right) / \gamma_{b}},
$$

where $p_{b}(r)$ is the partial pressure of buoyant gas inside bubbles at radius $r$ and $\gamma_{b}$ is the adiabatic index of buoyant gas. Assuming that the partial pressure scales with the thermal pressure of the ICM, the volume heating function $\mathcal{H}$ can be expressed as

$$
\mathcal{H} \sim-h(r) \nabla \cdot \frac{\dot{e}}{4 \pi r^{2}}=-h(r)\left(\frac{p}{p_{0}}\right)^{\left(\gamma_{b}-1\right) / \gamma_{b}} \frac{1}{r} \frac{d \ln p}{d \ln r},
$$

where $p_{0}$ is the central pressure and $h(r)$ is the normalization function

$$
h(r)=\frac{L}{4 \pi r^{2}}\left(1-e^{-r / r_{0}}\right) q^{-1}
$$

In equation (11), $L$ is the luminosity of the central source and $q$ is defined by

$$
q=\int_{r_{\min }}^{r_{\max }}\left(\frac{p}{p_{0}}\right)^{\left(\gamma_{b}-1\right) / \gamma_{b}} \frac{1}{r} \frac{d \ln p}{d \ln r}\left(1-e^{-r / r_{0}}\right) d r
$$

where $r_{0}$ is the inner heating cutoff radius. Interestingly, the cosmic ray heating model for cooling flows (Loewenstein et al. 1991) predicts a very similar functional form for the heating function. In this model, heating is due to cosmic rays, which are produced in the central active nucleus and are trapped by Alfvén waves in the cooling flow plasma. They also do work on the thermal gas as they propagate down the pressure gradient. Radio galaxies are likely to be intermittent on a time scale much shorter than the Hubble time, and possibly as short as $t_{i} \sim 10^{4}-10^{5}$ yr (Reynolds and Begelman 1997). The bubble rise speed would be comparable to the sound speed, which in turn is comparable to the dynamical speed. Therefore, the cooling timescale is much longer than the bubble rise timescale, and it is justifiable to treat feedback heating $L$ in a timeaveraged sense. Therefore, in equation (11) we assume that the luminosity is injected instantaneously and neglect any delay between central activity and heating of the ICM. We also assume that all energy generated in the center goes into heating. In principle some fraction of the energy could escape the cluster in the form of sound waves. However, they are likely to carry away only a fraction of the energy comparable to that which is dissipated (e.g., (Reynolds et al. 2002; Churazov et al. 2002)). The physical motivation for the inner heating cutoff arises from the finite size of the central radio source. Energy from such a source will start to dissipate at a finite distance from the cluster center. Unlike heating functions that depend on local microscopic physics, this heating function is nonlocal in the sense that it depends on the pressure gradient. In this regard, it resembles thermal conduction, but here the heating rate depends on the gradient of pressure rather than temperature. For simplicity and in order to avoid numerical complications, we adopt hydrostatic values to calculate the logarithmic derivative in the code (i.e., $\nabla p=-\rho \nabla \Psi)$.

In the specific case considered below, the feedback luminosity is assumed to be $L \sim-\epsilon \dot{M} c^{2}$, where $\dot{M}=4 \pi r_{\min }^{2} \rho v$ is the accretion rate at the inner radius $r_{\min }$ and $\epsilon$ is the accretion efficiency. 


\subsection{Numerical methods}

All of the calculations presented in this paper use the ZEUS-3D (Clarke et al. 1994) code in its 1D mode. The code has been modified by including a non-singular isothermal potential, feedback heating, cooling, convection and conduction. For stability, the convection and conduction terms have to be integrated using time steps that satisfy appropriate Courant conditions. The Courant conditions for conduction and convection read

$$
\begin{gathered}
\Delta t_{\mathrm{conv}} \leq \frac{1}{2} \frac{e(\Delta r)^{2}}{f \kappa T^{5 / 2}} \\
\Delta t_{\text {cond }} \leq \frac{1}{2} \frac{2^{5 / 2}}{\gamma} \frac{(\Delta r)^{5 / 2}}{g^{1 / 2} l_{m}^{2}} .
\end{gathered}
$$

Whenever either (or both) of these timescales is smaller than the time step used for hydrodynamic equations, we integrate the respective terms separately at the smaller time step. This method is analogous to the one used by (Stone et al. 1999) in hydrodynamical simulations of viscous non-radiative accretion discs.

Our computational grid extends from $r_{\min }=1 \mathrm{kpc}$ to $r_{\max }=200 \mathrm{kpc}$. In order to resolve adequately the inner regions it is necessary to adopt a non-uniform grid. We use a logarithmic grid in which $(\Delta r)_{i+1} /(\Delta r)_{i}=\sqrt[N-1]{10}$, where $N$ is the number of grid points. Our standard resolution is $N=400$.

\subsubsection{Initial and boundary conditions}

In order to generate initial conditions we solve the equation of hydrostatic equilibrium of a gas at constant temperature. We assume that the gas density initially constitutes a fraction $f_{\text {igas }}$ of the cluster dark matter density at the inner radius. The gas is assumed to be in contact with a pressure and thermal bath at the outer radius. Thus, we ensure that temperature and density at the outer radius are constant. We adopt inflow/outflow boundary conditions at the outer radius but at the inner boundary we use outflow boundary conditions. We extrapolate hydrodynamic variables from the active zones to the "ghost" zones used to compute the derivatives of the hydrodynamic variables. At the inner boundary we allow mass to flow out of the computational region $(v \leq 0)$ and use a switch to ensure that the fluid can only flow toward the center (i.e., boundary value of the velocity is zero if the gas tries to flow in the opposite direction). Feedback is assumed to be present only when the gas at the inner boundary flows inwards $(v<0)$.

\section{RESULTS}

To illustrate our method we now present one representative model. This model reproduces the main features of the observed cooling flows including a floor in the temperature at about $1 \mathrm{keV}$. We note that fine tuning is not required to obtain stable equilibrium solutions. Figure 1 shows the evolution of temperature (upper left panel), electron number density (upper right panel), entropy $\left(S \equiv k_{B} T / n_{e}^{\gamma-1}\right.$; lower left panel) and mass accretion rate as a function of distance from the cluster center. We follow cluster evolution for one Hubble time $t_{H}=H_{0}^{-1}\left(H_{0}=75 \mathrm{kms}^{-1} \mathrm{Mpc}^{-1}\right)$. Each curve within a given panel is plotted every $1 / 40$ or $1 / 100$ of the Hubble time, as specified in the caption. The initial gas temperature is set to be $4 \mathrm{keV}$ throughout the cluster. Specific parameters of the model presented in Figure 1 are as follows: $\epsilon=0.003, \gamma_{b}=4 / 3, \gamma=5 / 3, r_{0}=20 \mathrm{kpc}, r_{c}=30 \mathrm{kpc}$, $r_{g}=4 \mathrm{kpc}, \sigma_{g}=800 \mathrm{~km} \mathrm{~s}^{-1}, \sigma_{c}=300 \mathrm{~km} \mathrm{~s} s^{-1}, f=0.23$, $Z=0.3 Z_{\odot}$ and $f_{\text {igas }}=0.025$ (which corresponds to the final gas mass fraction $f_{\text {gas }}(r<200 \mathrm{kpc})=M_{\text {gas }}(<$ $\left.r) / M_{\text {tot }}(<r) \sim 0.06\right)$.

Line and free-free cooling in the cluster center leads to a slow decrease in temperature. At this stage feedback is not yet very important. The initial phase of slow cooling is followed by a gradual increase in cooling rate, which is caused mainly by the increase of density in the center. The feedback heating is controlled by the accretion rate in the center. Therefore, the cluster does not cool in runaway fashion. Once the average inflow velocity in the center has become sufficiently high and sufficient density has accumulated in the center of the cluster, the feedback becomes strong enough to suppress further decrease in temperature. The cluster then quickly relaxes to an equilibrium state. The evolution of gas density is similar. Initially, gas accumulates gradually in the center of the cluster. When feedback becomes strong, further accumulation is prevented and the central density is stabilized. Entropy and mass accretion rate exhibit a similar behavior - the former relaxes to a stable equilibrium and the latter, after oscillations through positive and negative values, tends to a small constant negative value as a function of radius. In the final state, the accretion rate at the inner radius is $\dot{M} \sim-1.76 M_{\odot} \mathrm{yr}^{-1}$, which is much smaller than acretion rates inferred from the standard cooling flow models. Our $\dot{M}$ corresponds to the bolometric feedback luminosity $L \sim 3 \times 10^{44} \mathrm{erg} \mathrm{s}^{-1}$. The most important aspect of the cluster evolution is that it is possible for the cluster to reach sustainable and stable equilibrium. In our model, there is no need for mass dropout distributed throughout the cooling flow region. However, some fraction of the material in the central regions may become thermally unstable and form $\mathrm{H} \alpha$ emitting filaments or stars (e.g., (Blanton et al. 2001; McNamara 2002)). Adding mass dropout terms corresponding to these effects would only improve the stability properties of our models. It could also decrease the central gas density and, thus, lead to a higher minimum temperature.

Figure 2 shows the temperature, electron number density, entropy and mass accretion rate as a function of time (in units of Hubble time $t_{H}=H_{0}^{-1}, H_{0}=75 \mathrm{~km}$ $\left.\mathrm{s}^{-1} \mathrm{Mpc}^{-1}\right)$ for different distances from the cluster center $r=5,10,20,40,80,160 \mathrm{kpc}$. The parameters are the same as for Figure 1, i.e., Figure 2 shows crosssections through the panels in Figure 1 at the above radii. In the case of entropy and temperature, the above sequence of $r$ corresponds to the curves from bottom to top. For density the trend is opposite. In the case of accretion rate, the amplitude of oscillations increases with $r$ $(=5,10,20,40,80)$. The oscillations are due to sound waves, which propagate across the cluster as it adjusts to changing conditions. The precise character of these sound waves, i.e., number of peaks per unit time, depends on the boundary conditions. As can be clearly seen, the slow initial evolution of the cluster is followed by a faster 
"collapse" stage around $t=0.2 t_{H}$. After this phase, the cluster stabilizes and reaches its final equilibrium state. The profiles shown in Figure 1 bear close resemblance to the observed temperature, density and entropy profiles (e.g., (Johnstone at al. 2002) Chandra observations of Abell 2199).

Although nominally present in the code, convection is not important for the parameters of the model presented in this paper. The intracluster medium usually does not become convectively unstable. We also considered models with stronger heating in the center (i.e., higher efficiency $\epsilon$ ). In such models the gradient of entropy was significantly negative and convection was present. However, strong heating prevented the gas from accreting in the center. This led to the accumulation of material and, subsequently, to a cooling catastrophe.

\section{CONCLUSIONS}

We have proposed a new class of time-dependent cooling flow models where cooling is offset by a combination of cen- tral AGN heating and thermal conduction from the outer regions. Our models do not require any mass dropout rate distributed throughout the cluster. We showed that it is possible to obtain stable final equilibrium states, which do not suffer from the cooling catastrophe. We have presented a representative model, which reproduces the main features of observed cooling flows, including a floor in the temperature at about $1 \mathrm{keV}$. We have also found stable models for other parameters, which we will present later. Fine tuning is apparently not required to obtain stable equilibrium solutions. Moreover, stable models are characterized by gas accretion rates that are much smaller than the mass dropout rates predicted by standard cooling flow models.

We are grateful to Phil Armitage, Fabian Heitsch, Christian Kaiser and Daniel Proga for helpful discussions and the referee for a fast response. This work was supported in part by NSF grant AST-9876887.

\section{REFERENCES}

Allen, S.W. et al. 2001, MNRAS, 324, 842

Begelman, M.C. 2001, in Gas and Galaxy Evolution, APS Conf. Proc., vol. 240, ed. Hibbard, J.E., Rupen, M.P., and van Gorkom, J.H., p. 363, (astro-ph/0207656)

Bertschinger E. \& Meiksin A., 1986, ApJ, 306, 1

Binney, J., \& Tabor, G. 1995, MNRAS, 276, 663

Blanton, E.L., Sarazin, C.L., McNamara, B.R., \& Wise M.W. 2001, ApJL, 558, 15

Brighenti, F., \& Mathews, W.G. 2002, ApJ, 573, 542

Churazov, E., Sunyaev, R., Forman, W., \&Böhringer, H. 2002, MNRAS, 332, 729

Clarke, T.E., Kronberg, P.P. \& Böhringer, H., 2001, ApJ, 547, 111

Clarke, D.A., Norman, M.L. \& Fiedler, R.A. 1994, ZEUS-3D users manual version 3.2.1, University of Illinois

David, L.P. et al. 2001, ApJ, 557, 546

Fabian, A.C., 1994, ARAA, 32, 277

Fabian, A.C., 2001, MNRAS, 321, 20

Fabian, A.C., Voigt, L.M., \& Morris, R.G. 2002, MNRAS, submitted, (astro-ph/0206437)

Johnstone, R.M., Allen, S.W., Fabian, A.C., \& Sanders, J.S. 2002 MNRAS, in press (astro-ph/0202071)

Jones, C. et al. 2002, ApJ, 567, 115
Kronberg, P.P., Dufton, Q.W., Li, H., \& Colgate, S.A. 2001, ApJ, 560,178

Loewenstein, M., Zweibel, E., \& Begelman, M.C. 1991, ApJ, 377, 392

Malyshkin, L. 2001, ApJ, 554, 561

McNamara, B.R. 2002, in X-rays at Sharp Focus: Chandra Science Symposium, ed. Vrtilek, S., Schlegel E.M., \& Kuhi, L., (astro-ph/0202199)

McNamara, B.R. et al. 2001, ApJ, 562, 149

Meiksin, A., 1988, ApJ, 334, 59

Narayan, R. \& Medvedev, M.V., 2001, ApJ, 562, 129

Peterson, J.R. et al. 2001, A\&A, 365, 324

Reynolds, C.S. \& Begelman, M.C., 1997, ApJ, 487, 135

Reynolds, C.S., Heinz, S., \& Begelman, M.C. 2002, MNRAS, 332, 271

Schmidt, R.S., Allen, S.W. \& Fabian, A.C. 2001, MNRAS, 327, 1057

Stone, J.M., Pringle, J.E., \& Begelman, M.C. 1999, MNRAS, 310, 1002

Sutherland, R.S., \& Dopita, M.A. 1993, ApJS, 88, 253

Tozzi, P., \& Norman, C. 2001, ApJ, 546, 63

Voigt, L.M., Schmidt, R.W., Fabian, A.C., Allen, S.W., \& Johnstone, R.M. 2002, MNRAS, in press, (astro-ph/0203312) 

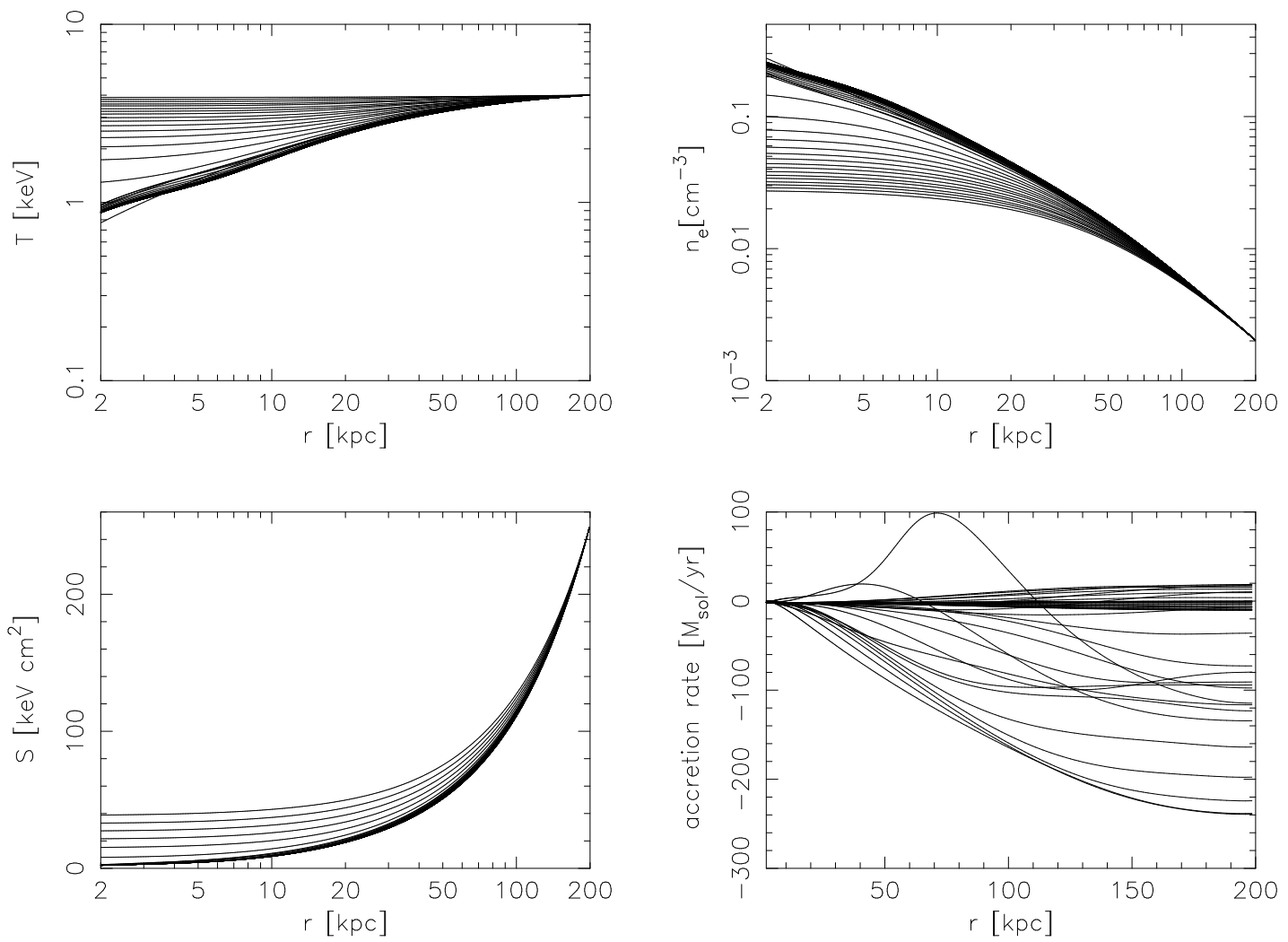

FIG. 1. - Time sequence of temperature (upper left panel), electron number density (upper right), entropy $\left(S \equiv k_{B} T / n_{e}^{\gamma-1}\right.$, lower left) and accretion rate. Temperature and density are shown every $0.01 H_{0}^{-1}$ and entropy and accretion rate every $0.025 H_{0}^{-1}$. The model settles down to a stable equilibrium state, which is visible via the dense concentration of curves. See text for additional information. 

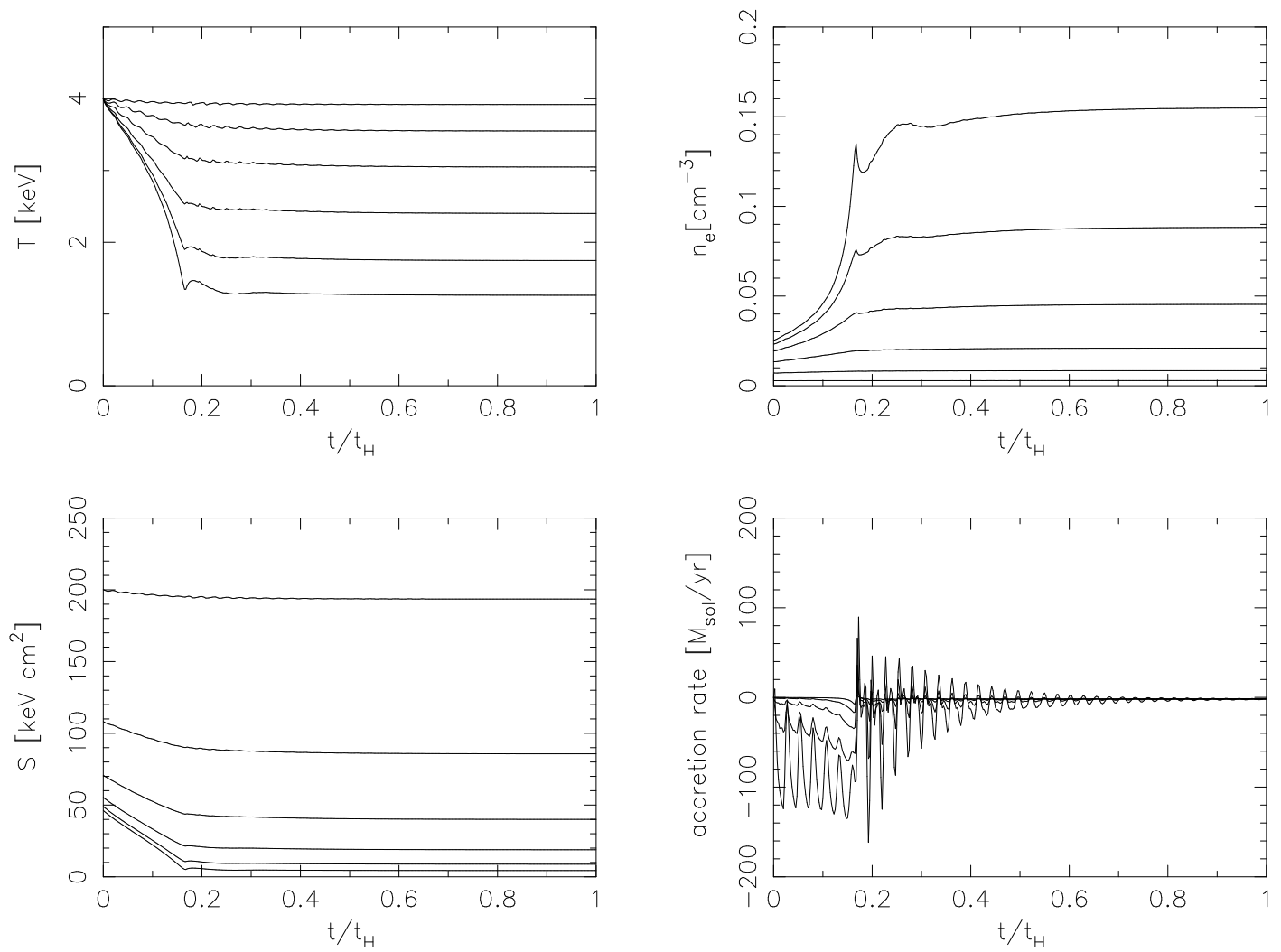

FIG. 2.- The dependence of temperature (upper left panel), electron number density (upper right), entropy (lower left) and accretion rate as a function of time for different distances from the cluster center. The final accretion rate is $\sim 1.76 M_{\odot} \mathrm{yr}^{-1}$. See text for details. 